\title{
Violencia y desarrollo social: más allá de una perspectiva de trauma*
}

Violence and social development: Beyond a trauma perspective

Recibido: marzo 24 de 2010 | Revisado: noviembre 19 de 2010 | Aceptado: enero 14 de 2011

\author{
ROBERTO POSADA GILÈDE ** \\ Carlos José Parales Quenza \\ Universidad Nacional de Colombia, Bogotá, Colombia
}

SICI: 2011-2777(201203)11:1<255:VYDSPT>2.0.CO;2-L

Para citar este artículo: Posada, R. \& Parales, C. J. (2012). Violencia y desarrollo social: más allá de una perspectiva de trauma. Universitas Psychologica, 11(1), 255-267.

* Los datos empíricos con población colombiana que soportan la tesis principal del artículo fueron obtenidos por el primer autor en el año 2003 con población en situación de desplazamiento forzado.

** Ciudad Universitaria. Carrera 30 \# 45-03, Ed. 212 (Ciencias Humanas), Of. 220, Tel.: 316500 Ext. 16345.E-mails: roposadagi@unal.edu.co,cparalesq@unal.edu.co

\section{RES UMEN}

Es ampliamente conocido que la exposición a violencia tiene consecuencias en la salud mental de los individuos. Sin embargo, un gran número de personas que crecen en medio de contextos violentos no muestran problemas psicológicos. La evidencia que presentamos en el presente artículo, sugiere que las experiencias de violencia no pasan desapercibidas y que, por el contrario, impactan el desarrollo social de los individuos y más específicamente el razonamiento moral. Las implicaciones de abordar el problema desde una perspectiva diferente a modelos de trauma, permiten explorar nuevos aspectos relacionados con la perpetuación de ciclos de violencia.

Palabras clave autores:

Desarrollo social, violencia, razonamiento moral, adversidad, salud mental. Palabras clave descriptores:

Bienestar psicológico, cognición social, revisión empírica.

\section{A B S T R A C T}

It is widely known that exposure to violence affects the mental health of individuals and groups. However, a large number of individuals who grow up in contexts of violence do not exhibit symptoms of mental disorder. Evidence presented in the article suggests that individual's experiences of violence do not go unnoticed, and on the contrary, have an impact on their social development and more specifically on their moral reasoning. The implications of the psychosocial perspectives for the study of the psychological consequences of violence, beyond trauma models, allow us to explore key aspects related to the perpetuation of cycles of violence.

Key words authors:

Social development, violence, moral reasoning, adversity, mental health.

Key words plus:

Welfare psychological, social cognition, empirical review. 
La violencia, en sus distintos tipos, es uno de los mayores males que aflige actualmente a la humanidad. Tanto los llamados "actos de terrorismo" alrededor del mundo que dan a conocer ahora constantemente los medios de comunicación, como las respuestas estatales, la ubicuidad de conflictos armados, así como altos índices de delincuencia y criminalidad, han ido generando un creciente interés en cómo estas experiencias se relacionan con el bienestar psicológico de las personas.

Crecer en contextos de violencia tiene indudablemente efectos en el bienestar psicológico. Diversos estudios muestran las consecuencias de la exposición a violencia en la salud mental (e.g., Fremont, 2004; Horn \& Trickett, 1998; Jenkins \& Bell, 1997; Palacio, Sabatier, Abello \& Madariaga, 2001). La violencia daña el tejido de la sociedad, erosiona el capital social y la eficacia colectiva, afectando de esta manera determinantes sociales de salud (Franco, 1997). En un estudio sobre las relaciones entre capital social y salud mental en una comunidad pobre de Colombia, Harpham, Grant y Rodríguez (2004) encontraron que la exposición a violencia y variables sociestructurales como género y empleo, fueron los principales determinantes de la situación de salud mental en esa comunidad.

Un buen número de investigaciones aborda las consecuencias psicológicas de la violencia con referencia a la salud mental, relacionándolas con la presencia de trastorno mental y enfatizando con frecuencia síntomas asociados al estrés postraumático (e.g., Echenique, Medina, Medina \& Ramírez, 2008; Londoño et al., 2005; Pérez-Olmos, Fernández-Piñeres \& Rodado-Fuentes, 2005; Rodríguez, De La Torre \& Miranda, 2002). Las secuelas de crecer en contextos adversos para el desarrollo social han sido menos estudiadas; si bien uno de los aspectos que probablemente es más vulnerable a condiciones de adversidad crónica como la violencia, son los entendimientos sociomorales de quienes crecen en esos contextos -la interpretación y evaluación- y, en términos más generales, el conocimiento y formas de pensar o teorías que las personas construyen acerca del mundo social, los cuales abarcan no solo a sí mismo, sino también a otros, a las relaciones con otros y a los sistemas institucionalizados de interacción social (Alvarado, Ospina \& Luna, 2005; Echavarría Grajales \& Vasco Montoya, 2006; Posada \& Wainryb, 2008; Quintero Mejía \& Vasco Montoya, 2007).

Aunque se ha asumido que los individuos que viven en contextos violentos aceptan o aprueban el uso de medios agresivos para resolver conflictos, lo cual reproduce ciclos de violencia, es importante examinar evidencia sobre los modos en los que las personas razonan acerca de situaciones de conflicto interpersonal, pues es indudable que estos sistemas de representación orientan las acciones individuales. También se deben considerar las maneras como los individuos coordinan necesidades y emociones con conceptos de bienestar y justicia, y los modos en los que experiencias de violencia se relacionan con representaciones del otro e influyen en el establecimiento de vínculos interpersonales y resolución de conflictos.

En este artículo presentamos una revisión de evidencia empírica que muestra como las experiencias de violencia, más allá de la presencia (o no) de síntomas o trastornos psicológicos, están asociadas a características particulares del conocimiento social de los individuos, resaltando que la configuración de relaciones sociales se encuentran interrelacionadas con el entendimiento moral y constituyen además una dimensión fundamental del bienestar psicológico (cf. Keyes, 2003). Sin pretender desconocer el alcance de estudios basados en modelos de trauma, destacamos la importancia de comprender cómo crecer en medio de experiencias adversas altera la constitución de relaciones sociales y afecta los vínculos de solidaridad. La relevancia de centrarse en la dimensión sociomoral está relacionada con la posibilidad de considerar estrategias que ayuden a romper ciclos de violencia, enfatizando nociones como responsabilidad social, cohesión y ciudadanía, y su promoción en la comunidad.

Para iniciar se presentan resultados de varios estudios que se centran en las consecuencias psicológicas de vivir en contextos de adversidad, para adentrarse luego en los efectos sobre el desarrollo social y, más específicamente, sobre el desarrollo moral. La exposición de este segundo tema se divide en tres partes: (1) presentación crítica de resultados 
de estudios realizados desde diferentes perspectivas teóricas, sobre la relación entre exposición a violencia y conocimiento moral; (2) fundamentos del enfoque de dominios específicos, como una aproximación alternativa que puede explicar de manera más adecuada la relación existente entre experiencias individuales y conocimiento sociomoral y (3) resultados de estudios empíricos, desde la perspectiva de los dominios específicos sobre las relaciones entre exposición a violencia y razonamiento moral, realizados en Colombia por uno de los autores.

\section{Efectos psicológicos de la exposición a violencia}

El número de estudios sobre los efectos de la exposición a violencia en la salud psicológica es abundante (e.g., Buckner, Beardslee \& Bassuk, 2004; Herrman, Saxena \& Moody, 2005) e incluye reportes sobre condiciones de salud mental en contextos de violencia sociopolítica crónica (e.g., Laplante \& Rivera Holguín, 2006; Mansour, 2002). Un buen número de estudios ha explorado los efectos negativos de la exposición a violencia en niños y adolescentes en términos de síntomas y trastornos del comportamiento -internalizadores y externalizadores- (e.g., Buka, Stichick, Birdthistle \& Earls, 2001; Gorman-Smith \& Tolan, 1998; Macksoud \& Aber, 1996, Martinez \& Richters, 1993; Osofsky, Wewers, Hann \& Fick, 1993; Palacio et al., 2001; Punamäki, 1996; Pynoos, Frederick, Nader \& Arroyo, 1987).

Los resultados de investigaciones realizadas en los Estados Unidos indican que la exposición a violencia está asociada con síntomas de estrés postraumático, trastornos internalizadores del comportamiento - usualmente, depresión y ansiedad-y trastornos externalizadores -habitualmente, agresividad y delincuencia- (Buka et al., 2001; Fitzpatrick, 1993; Gorman-Smith \& Tolan, 1998; Lynch, 2001; Miller, Wasserman, Neugebauer, Gorman-Smith \& Kamboukos, 1999; Pynoos et al., 1987; Richters \& Martinez, 1993). El maltrato familiar se considera un problema frecuente en comunidades violentas y los menores que reportan ser víctimas de este, usualmente manifiestan trastornos externalizado- res (Lynch \& Cicchetti, 1998). Esto significa que los menores de edad que viven en países que experimentan conflictos armados, especialmente por tiempo prolongado, tendrían más probabilidades de sufrir violencia doméstica y así mismo más probabilidades de manifestar trastornos externalizadores del comportamiento.

Por otra parte, los estudios también muestran que existen diferencias de género y edad. Las investigaciones han encontrado que entre los menores de edad que han experimentado violencia, las niñas tienden a reportar niveles más altos de estrés postraumático, depresión y trastornos internalizadores del comportamiento; mientras que los niños reportan niveles más altos de impulsividad, agresión y síntomas de trastornos externalizadores (véanse Jenkins \& Bell, 1997). Aunque en algunos casos no se han encontrado evidencias de relación entre exposición a violencia y agresividad (e.g., Macksoud \& Aber, 1996), la mayoría de estudios sí han reportado dicha asociación, incluyendo comportamientos delincuenciales (Barkin, Kreiter \& DuRant, 2001; Farver \& Frosch, 1996; Miller et al., 1999). Varios estudios también han mostrado que los adolescentes de más edad -generalmente, entre 15 y 18 añosreportan más experiencias de violencia que niños más jóvenes -ordinariamente, entre 9 y 14 años-y en algunos, aun que adultos jóvenes -entre 21 y 24 años- (Buka et al., 2001; Franco, 1997; Kuo, Mohler, Raudenbush \& Earls, 2000; Selner-O'Hagan, Kindlond, Buka, Randenbush \& Earls, 1998). Estos mismos estudios sugieren que los menores de edad de sexo masculino reportan más exposición a violencia que los de sexo femenino.

No todos los estudios que han explorado la pregunta acerca de la posible relación entre agresividad/comportamiento antisocial y exposición a violencia han encontrado los mismos resultados. Ninguno ha explorado las razones detrás de los actos agresivos y concretamente el razonamiento moral sobre tales actos. Además, la mayoría de estos estudios se han llevado a cabo en localidades violentas de los Estados Unidos y no en países que experimentan conflictos armados, lo cual puede influir no solo en los tipos de violencia experimentada, sino también en los significados 
y las comprensiones de dichas situaciones. Esos significados, y en general entendimientos, que se tengan de situaciones interpersonales informan los juicios morales y consecuentemente las decisiones sobre el comportamiento, por lo que el estudio de las consecuencias psicológicas de la adversidad, principalmente la violencia, necesariamente debe enmarcarse en el contexto sociocultural.

\section{Investigación sobre el desarrollo moral en contextos violentos}

La investigación sobre el desarrollo moral de menores de edad en países violentos es escasa y los pocos estudios publicados, desde diferentes perspectivas teóricas, han arrojado resultados mixtos. Dos de las perspectivas más reconocidas, la del aprendizaje social y la del desarrollo moral de Kohlberg, muestran limitaciones conceptuales y metodológicas. Desde el aprendizaje social, se plantea que el desarrollo moral gira en torno a la internalización de reglas consensuales y estándares. Consecuentemente, los consensos son necesarios para que los individuos respeten los derechos de otros y actúen justamente (Bandura, 1991). Esos estándares son trasmitidos o enseñados a los menores por medio del modelamiento, las sanciones y la información. Los humanos aprenden a través de la instrucción directa e indirecta, la imitación y los castigos y recompensas. Una vez los estándares son internalizados, mecanismos autorregulatorios producen la conducta moral (Bandura, 1991; Bandura, Barbaranelli, Carpara \& Pastorelli, 1996). Desde esta perspectiva, se podría esperar que los menores de edad que viven en países en situación de conflicto armado y, más específicamente, en medio de la violencia, aprendan comportamientos violentos como formas válidas de resolver conflictos, imiten conductas violentas de las cuales han sido testigos y generalicen esos comportamientos violentos a otros contextos sociales.

La investigación desde esta perspectiva correlaciona la observación de los comportamientos agresivos con exposición a violencia (e.g., Liddell, Kvalsvig, Qotyana \& Shabalala, 1994). En general, los estudios han encontrado que la presencia de violencia en la comunidad está asociada con comportamientos agresivos. Los menores que viven en áreas más violentas están más involucrados en situaciones agresivas, especialmente cuando tienen amigos mayores. Sin embargo, no se ha encontrado evidencia sobre la generalización de estos comportamientos a través de contextos.

Aunque esta perspectiva sugiere que hay asociación entre crecer en contextos violentos y agresividad, centrarse exclusivamente en el comportamiento no es suficiente para sacar conclusiones acerca de la aprobación o aceptación de medios violentos para resolver conflictos interpersonales, ni mucho menos sobre el desarrollo moral. Otros aspectos como las intenciones, las emociones y, en general, el razonamiento, son necesarios para definir lo moral. Además, medir comportamientos agresivos sin especificar el tipo de situaciones en las cuales las conductas agresivas aparecieron, hace difícil concluir sobre cómo los menores pueden concebir e interpretar esas situaciones concretas.

En otros estudios en los que sí se tomó en cuenta el razonamiento, aunque este no fuera el objetivo principal, encontraron que los niños que han experimentado violencia relacionada con actos de guerra, usualmente recurren a conceptos morales y prosociales. Por ejemplo, Punamäki (1996) encontró que niños israelíes expresaban preocupaciones morales (i.e., dilemas) cuando evaluaban la guerra.

Por otra parte, Coles (1986) encontró que niños que han crecido en contextos de conflicto político, expresan deseos de ayudar a otros que han estado en situaciones de victimización similares a las que ellos han experimentado. De acuerdo con Coles, estos niños desarrollan una sensibilidad moral avanzada y precoz. Nuevamente, al igual que en el estudio de Punamäki (1996), y a pesar de las diferentes conclusiones, ambas investigaciones muestran que los conceptos morales están presentes en el razonamiento de los menores acerca de situaciones de conflicto y violencia, y que sus experiencias de violencia previa parecen estar relacionadas con su razonamiento sociomoral. Sin embargo, ninguno de estos estudios evaluó directamente el razonamiento moral.

La mayoría de estudios sobre razonamiento moral de menores en países con conflictos armados, 
han sido conducidos desde perspectivas constructivistas, basadas principalmente en la Teoría de Kohlberg (e.g., Cairns, 1987; Ferguson \& Cairns, 1996). Desde esta teoría, los conceptos morales autónomos o basados en principios universales se desarrollan a partir de conceptos heterónomos. En esta perspectiva, los conceptos morales tempranos se fundamentarían en consideraciones de autointerés, evitación de castigo y seguimiento de convenciones sociales. Es solo hasta la adolescencia, cuando los conceptos morales autónomos llegarían a diferenciarse, sustituyendo esa incipiente moralidad basada en la conformidad y la obediencia.

Kohlberg (1976) afirmaba, además, que los ambiente ricos en oportunidades de toma de perspectiva del otro, son importantes para la estimulación del desarrollo moral. Es importante notar que en contextos de violencia continua y persistente, el pensamiento de las personas probablemente está polarizado y, así, con pocas oportunidades para tomar la perspectiva de otros. En ese sentido, uno podría esperar que desde una perspectiva kohlbergiana los menores que han crecido en medio de la violencia, estén en un nivel más bajo de razonamiento moral, comparados con aquellos que viven en áreas o comunidades no violentas. Sin embargo, también es cierto que en contextos de deprivación y adversidad, la posibilidad de formar representaciones de segundo orden y así asumir la perspectiva del otro, constituye una ventaja estratégica que permite la supervivencia y la ganancia individual. Por lo tanto, nuestra hipótesis es que en contextos de desventaja social y violencia, la posibilidad de representarse la perspectiva del otro no solo es posible, sino también necesaria. El problema estaría en reconocer cómo el contexto valida las formas y propósitos en que las perspectivas de otros son tenidas en cuenta.

En contextos de fractura de los vínculos sociales, caracterizados por desconfianza y aislamiento social, es probable que la perspectiva del otro se tenga en cuenta de manera completamente egoísta con desconocimiento del entorno, con el fin de obtener ventajas en situaciones de interacción. Esa forma paradójica de reconocimiento del otro no es útil para la cooperación social y el desarrollo de la confianza.
La mayoría de estudios con poblaciones que han experimentado conflicto armado y que han adoptado la perspectiva de Kohlberg, se han centrado en categorizar a los menores a lo largo del continuo de etapas propuestas en la teoría, con resultados mixtos (e.g., Cairns, 1987; Ferguson y Cairns, 1996; Fields, 1973 citado por Dawes, 1994). Algunos de estos estudios han encontrado que los menores de países en situación de conflicto (e.g., Irlanda del Norte), muestran un razonamiento moral inferior a los que habitan en países que no experimentan conflictos armados (e.g., Fields, 1973 citado por Dawes, 1994). Por otro lado, otros estudios con poblaciones similares a las del estudio anterior no han encontrado diferencias y, en algunos casos, han mostrado tendencias a una mayor desaprobación de actos violentos en menores que han crecido en contextos de conflicto armado (Lorenc \& Branthwaite, 1986).

En general, estos estudios presentan limitaciones de consideración. Por un lado, no evaluaron cuidadosa y detalladamente la exposición a violencia. Generalmente, se asumió que por vivir en las mismas zonas, los participantes habían experimentado los mismos tipos de violencia. Es poco probable que todos hayan experimentado el mismo tipo y cantidad de situaciones violentas, además de que las interpretaciones y las vivencias subjetivas varían de acuerdo con la historia individual (e.g., Posada \& Wainryb, 2008).

Por otro lado, los estudios desde la perspectiva de Kohlberg han valorado el desarrollo moral de los niños sin tomar en cuenta los contextos particulares, asumiendo que el razonamiento moral es homogéneo a través de contextos y condiciones. Esta idea de la homogeneidad en el desarrollo moral a través de contextos sociales ha sido cuestionada durante los últimos 35 años por investigadores que adoptan el enfoque de los dominios específicos (cf. Turiel, 1998).

\section{Conocimiento moral: un enfoque de dominios específicos}

A diferencia de los enfoques por etapas del desarrollo moral, la propuesta del Enfoque de los Dominios ha sido la de que el conocimiento social es, desde 
un comienzo, organizado alrededor de diferentes dimensiones de pensamiento. Se han definido, por lo menos, tres dominios a saber: moral, personal y convencional (Turiel, 1983). Comenzando a una edad muy temprana, los niños construyen conceptos morales (dominio moral), a partir de interacciones que tienen características moralmente relevantes (e.g., situaciones en las cuales hay una víctima indispuesta que es lastimada o tratada injustamente). A la vez, los niños construyen también conceptos que tienen que ver con metas y jurisdicción personal (dominio personal), así como también con convenciones sociales y expectativas (dominio convencional).

Por lo tanto, desde este enfoque, incluso los niños pequeños desarrollan conceptos morales que se fundamentan no en consideraciones de castigo, autoridad o metas egoístas, sino en preocupaciones genuinas por el bienestar de otras personas y por la justicia de la situación. Un gran número de estudios ha apoyado la hipótesis del enfoque de los dominios específicos, tanto en sociedades occidentales como en no occidentales y han demostrado que, comenzando a la edad de aproximadamente 4 años, los niños evalúan los actos que son intencionalmente lastimadores o injustos como moralmente equivocados y justifican sus juicios usando conceptos basados en aspectos de justicia y bienestar (para revisiones amplias sobre este enfoque, véanse Smetana, 1995; Turiel, 1998).

Sin embargo, la idea de diferentes dominios o terrenos del pensamiento no significa que la aplicación de los conceptos morales sea homogénea. El enfoque de los dominios específicos también concibe que la aplicación de los conceptos morales a situaciones concretas varíe. Diversas fuentes de variación en la aplicación de conceptos morales han sido encontradas. Una de ellas es la interpretación subjetiva de la situación. En una serie de estudios se ha encontrado que las personas pueden compartir el mismo concepto moral, pero evalúan la situación de manera diferente o toman decisiones diferentes debido a la manera en que interpretan o conciben la situación (e.g., Shaw \& Wainryb, 1999; Wainryb, 1991, 1993; Wainryb \& Ford, 1998; Wainryb, Shaw \& Maianau, 1998).
Otras investigaciones han encontrado que los individuos pueden aplicar sus conceptos morales diferencialmente dependiendo de los tipos de asuntos o consideraciones que implique la situación que se evalúa, de manera que lo moral se resuelve y justifica en términos de la implicación de la acción (Helwig, 1995, 1997, 1998). Por ejemplo, Helwig (1995, 1997) encontró que niños y adolescentes que habían construido conceptos morales concernientes a derechos civiles, no los aplicaban consistentemente a situaciones de conflicto que derivaban en lastimar o que implicaban desigualdad. De manera similar, Posada y Wainryb (2008) encontraron que tanto niños como adolescentes en situación de desplazamiento que habían crecido en medio de violencia, pobreza e ilegalidad y que en abstracto evaluaban como incorrecto robar y lastimar a otros, aplicaban sus conceptos morales de manera diferente dependiendo de la situación. Por ejemplo, en una situación hipotética de extrema necesidad, evaluaron como malo robar y/o causar daño a otros para obtener lo que necesitaban. Sin embargo, en una situación de revancha contra alguien que los había lastimado previamente, muchos de ellos evaluaron como correcto robar y/o herir a esa persona. Por lo tanto, la necesidad de recuperar el equilibrio moral causado por el daño sentido justifica la acción, desde el marco de características culturales que promueven la retaliación como respuesta apropiada en determinadas circunstancias.

La gran mayoría de estudios realizados desde el enfoque de los dominios específicos, se han llevado a cabo con poblaciones que viven en condiciones normativas (esto es, regulares). Estudios realizados con preescolares víctimas de abuso (Smetana, Kelly \& Twentymen, 1984), niños agresivos (Astor, 1994; Astor \& Behre, 1997) y adolescentes delincuentes (Tisak \& Block, 1990), han mostrado que esos menores también desarrollan conceptos morales. A la vez, los resultados de esos estudios, con respecto a las formas en que los menores aplican conceptos morales a situaciones específicas relevantes para cada grupo, sugieren que estos aplican sus experiencias específicas al razonamiento moral (e.g., los razonamientos de menores abusados tienden a resaltar instancias de maltrato físico; el razonamiento 
de niños agresivos tiende a resaltar instancias que involucran provocación y retribución).

Los menores colombianos en situación de desplazamiento, que han crecido en medio de violencia e injusticia social, constituyen una clase diferente de población con condiciones no normativas. Es probable que algunos de estos menores muestren alteraciones que correspondan a algunas de las categorías de trastorno mental existente, tales como estrés postraumático, problemas de ansiedad, depresión y trastornos externalizadores del comportamiento (cf. Internal Displacement Monitoring Centre, 2008; Médecins Sans Frontières, 2006; Palacio et al., 2001). Pero no hay razones para suponer que esta población constituye una excepción a los resultados generales de los estudios a los que nos referimos en el párrafo anterior.

En el estudio de Posada y Wainryb (2008) se encontró que los menores colombianos en situación de desplazamiento han desarrollado conceptos morales basados en justicia y preocupación por el bienestar de los otros. Esto no quiere decir que no hayan sido afectados por las experiencias de violencia e injusticia social que han vivido. $\mathrm{Al}$ igual que otros estudios sobre cognición social con poblaciones en situaciones adversas, ese estudio mostró que existen características particulares en la forma de razonar acerca de situaciones conflictivas. Se han llevado a cabo estudios sobre las consecuencias de la exposición a violencia en la cognición social, los cuales merecen una revisión, en cuanto a sus principales resultados e implicaciones.

\section{Experiencias de violencia y cognición social}

Como afirma Turiel (1983) el conocimiento social se deriva de las interacciones con otros. Así, es de esperar que vivir en medio de la violencia esté asociado a tipos de conocimientos particulares que las personas construyen sobre la naturaleza social, sobre el otro y las condiciones del mundo en el que viven. Hasta la fecha, no existen muchos estudios sobre los efectos de la violencia continua en el desarrollo de la cognición social, pero sin duda las relaciones sociales violentas afectan las formas de solidaridad y las representaciones de ideas de justicia, confianza, responsabilidad y compasión.

Estudios con menores que crecen en medio de violencia han encontrado, por una parte, que estos menores utilizan pocas explicaciones psicológicas cuando hablan acerca de conflictos interpersonales (Fonagy, 2003; Posada, 2008; Sanderson, 2001; Walton \& Brewer, 2001). Se ha sugerido que los menores reflejarían en sus narrativas desconocimiento de los estados mentales de otros, de sus posibles creencias, intenciones, emociones, gustos y deseos, cuando se les pide hablar sobre situaciones de conflicto interpersonal (e.g., Fonagy, 2003). No obstante, la idea de la carencia de capacidad para reconocer estados mentales en situaciones interpersonales, necesita más evaluación empírica, ya que existen factores que pueden intervenir para que estos elementos sean tenidos en cuenta o no, y/o sean relevantes o no, a la hora de interpretar la situación. Por ejemplo, un factor fundamental al analizar una narrativa de conflicto interpersonal es la perspectiva del individuo en el conflicto, es decir, si fue la víctima o el victimario (Baumeister, 2000; Posada, 2008; Wainryb, Brehl \& Matwin, 2005).

Desde la perspectiva de víctima, comparado a la de victimario, no se suele utilizar tan a menudo información sobre los estados mentales de otros (como por ejemplo intensiones o emociones del victimario), pero desde la perspectiva de victimario, se da relevancia a las emociones de la víctima, sin dejar de lado las propias intenciones y emociones, además de ver al otro muchas veces como un provocador, lo cual justifica la acción para el sujeto. De esta manera, se ha mostrado, que no es cuestión de déficits o falta de habilidad para considerar los estados mentales propios o de los demás, sino que se está sesgado, por una parte, en función de la perspectiva y, por otra, debido a las experiencias sociales que se han tenido. Estos sesgos que se presentan desde cada perspectiva parecen alimentar ciclos de agresiones, puesto que al no considerar como relevante cierta información, como los estados mentales (e.g., las intenciones o las emociones del otro), o ver al otro como un provocador en un medio donde los grados de impunidad son altos, 
deja como información principal de la situación a evaluar los actos lastimadores.

De esta manera, si los actos lastimadores son vistos como intencionales y sin razones aparentemente justas, o buscando un beneficio solamente personal y egoísta, lo poco que le queda a los individuos en la mayoría de casos es defenderse o tratar de restaurar la justicia a través de un acto de retaliación.

Por otra parte, estudios con menores de edad que crecen en contextos de violencia han explorado la posible asociación entre exposición a violencia y la aceptación de medios agresivos para solucionar conflictos. En un estudio, con una muestra de niños habitantes de Chicago y en localidades con diferentes niveles de violencia, Guerra, Huesmann y Spindler (2003) encontraron que la exposición a violencia estaba relacionada no solo con niveles de agresividad más altos, sino también con pensamientos normativos acerca de la agresividad y con fantasías agresivas. Estos investigadores concluyeron que los menores de edad que viven en contextos violentos, desarrollan esquemas cognitivos que los hace ver el mundo como hostil y las respuestas agresivas como algo aceptable. Lo que explica, según estos autores, que los menores expuestos a violencia sean más agresivos es que han codificado en la memoria más guiones agresivos para resolver conflictos. La violencia, por lo tanto, se vuelve una respuesta normal para relacionarse con un mundo amenazante y peligroso.

Aunque se ha hallado que las personas que han sido expuestas a violencia aceptan respuestas agresivas como forma de solucionar conflictos, otros estudios han mostrado que no se pueden generalizar estos resultados a través de todas las situaciones, sino que es importante especificar los contextos en los que los individuos juzgan ese tipo de respuestas positivamente (e.g., Posada, 2008, 2009; Posada \& Wainryb, 2006, 2008).

En estudios llevados a cabo por Posada, se encontró que la aplicación de los conceptos morales no es homogénea a través de distintos contextos. En uno de esos estudios (Posada \& Wainryb, 2008), menores de edad colombianos en situación de desplazamiento fueron entrevistados con el fin de explorar la aplicación de conceptos morales en situaciones hipotéticas, donde robar y lastimar a otros podían ayudar con aspectos de sobrevivencia o con cumplir funciones de retaliación. Los resultados mostraron que la casi totalidad de los menores evaluaron como incorrecto robar o lastimar a otros en condiciones de extrema necesidad (condición de sobrevivencia), justificando sus juicios con razones concernientes a justicia y bienestar. Sin embargo, en una condición de revancha, un número significativo evaluó como correcto (esto es, una evaluación positiva) robar y lastimar a otros y únicamente una minoría de aquellos que hicieron una evaluación negativa, la justificaron con conceptos que tuvieran que ver con justicia y/o bienestar de los otros (es decir, con razones morales).

Estos menores justificaron sus juicios en la condición de revancha con razones relacionadas con prudencia, evitación de castigo y convenciones sociales (por ejemplo, evitar que se venguen con la familia o que lo metan a cárcel, las cuales son razones pertenecientes a los dominios personal y convencional). Por lo tanto, en este contexto, preocupaciones por asuntos de venganza contra aquellos que los han lastimado a ellos mismos y a sus familias parecen tener prioridad.

Otro resultado relevante de aquel estudio es que la mayoría de los menores hicieron una distinción entre lo que debería ser (nivel prescriptivo) y lo que probablemente sucedería si se presentara realmente la situación. A los participantes se solicitó que terminaran una historia antes de que el entrevistador les contara el final. Aunque los menores juzgaron como incorrectos los actos de robar o maltratar a otros cuando el entrevistador les presentó esas alternativas como finales de la historia, previamente cuando ellos expusieron sus expectativas, preveían que la situación iba a terminar generalmente en robo. Esto parece indicar que estos menores no tienen expectativas de un mundo justo y de que las cosas no son como deberían ser y que no se puede ser consecuente con lo que se considera correcto.

Otro hallazgo para resaltar aquí es que los adolescentes evaluaron los actos de venganza de forma más positiva que los niños (Posada \& Wainryb, 2008). De esta manera, surgió la pregunta sobre si las evaluaciones sociomorales estaban relacionadas con características específicas de la exposición 
a violencia, ya que fue este grupo de edad el que reportó una mayor exposición a eventos violentos.

Con el propósito de investigar este asunto, se analizó la relación existente entre las valoraciones de menores desplazados sobre actos lastimadores o injustos en situaciones hipotéticas de extrema necesidad, de posibilidad de revancha y cuando ninguna de estas consideraciones estaba presente, y el autorreporte de exposición a violencia de cada participante (Posada, 2009; Posada \& Wainryb, 2006). Específicamente, se examinó si haber estado expuesto crónicamente a violencia, y particularmente como testigo, estaba relacionado con la aprobación de actos como robar y lastimar a otros en contextos de extrema necesidad y venganza. También, dentro de estos mismos contextos, se examinaron posibles asociaciones entre el grado de proximidad de víctima y victimario (es decir, si eran personas cercanas -familiares, amigos-o simplemente conocidos), en las diversas experiencias de violencia de los participantes, y las justificaciones de los juicios morales sobre aquellos actos que incluían robo o maltrato físico (en situaciones hipotéticas).

Los resultados mostraron, primero, que la exposición a violencia está relacionada con los juicios morales en un contexto de venganza. Los menores que reportaron más exposición a violencia como testigos evaluaron más positivamente lastimar a otros. Estos mismos participantes justificaron sus juicios morales, más a menudo, con razones que incluían pensamientos de retaliación y menos aspectos relacionados con convenciones sociales, personales y pragmáticas, razones pertenecientes a dominios no morales. Es decir, aceptaban la utilización de medios violentos como una forma de restaurar la justicia y no como un medio aceptable bajo cualquier circunstancia. Los menores de edad que habían sido testigos de más actos violentos en contra de miembros de su familia, tendieron a justificar sus juicios morales con razones basadas en retaliación.

Diversos estudios sobre el desarrollo social en medios violentos han mostrado que la exposición a violencia los impacta significativamente. Sesgos específicos con respecto a la información que se tiene en cuenta en situaciones de conflicto interpersonal, están relacionados con la manera en que se evalúan situaciones y muy probablemente con decisiones que se toman. Es importante resaltar que aunque en este tipo de poblaciones generalmente se presentan mayores niveles de agresividad $\mathrm{y}$ violencia, estudios recientes sugieren que esto no se debe a que consideren la violencia como un medio adecuado o aceptable moralmente y el cual debe ser usado en la mayoría de ocasiones o en cualquier situación. Por el contrario, la consideran indeseable pero piensan en la venganza como un medio efectivo para restaurar justicia.

\section{Conclusión}

Crecer en medios adversos y especialmente caracterizados por violencia, tiene implicaciones profundas en el desarrollo y funcionamiento psicológico, incluyendo formación de subjetividad, perspectivas de mundo, relaciones sociales y bienestar psicológico. En sociedades en las que se presentan conflictos armados, especialmente prolongados, la violencia se suele convertir en un problema generalizado que afecta al conjunto de la sociedad. Aunque aquellos que experimentan situaciones violentas extremas suelen presentar alteraciones en la salud, un número importante de individuos no presentan condiciones clínicamente significativas. Sin embargo, esto no quiere decir que tales individuos no han sido afectados psicológicamente por experiencias de adversidad.

$\mathrm{Al}$ igual que en condiciones normativas, los menores que han crecido en medios violentos desaprueban robar y lastimar a otros, tanto en abstracto como en contextos de necesidad económica. Sin embargo, al razonar acerca del mundo en el que viven consideran que la gente no se guía por estos principios para actuar. La distinción que hacen entre lo que debería ser y lo que realmente pasa en su mundo (lo cual es una característica del desarrollo que surge más adelante en poblaciones con condiciones normativas), parece afectar la motivación para actuar moralmente.

Además, los menores que han experimentado situaciones de adversidad extrema (como en el caso de los menores en situación de desplazamiento forzado) y que viven en un país en donde el sistema 
legal es incapaz de controlar y castigar a fuerzas al margen de la ley, consideran los medios agresivos como métodos adecuados para restablecer la justicia en situaciones donde ellos y/o sus familias han sido lastimados.

Lo anterior, sumado a que cuando hacen parte de un conflicto interpersonal pasan por alto las razones para actuar de los otros, o se sienten atacados y buscan defenderse o restaurar justicia, hace que surja la pregunta por su sentido como agentes morales. Es decir, a pesar de que tienen claro que robar y lastimar a otros es moralmente incorrecto, sienten que la realidad no les permite guiarse por sus concepciones de lo que debería ser. Esta discrepancia, estaría relacionada, por un lado, con la falta de motivación para confiar en los otros y con su habilidad para controlar la agresividad, ser solidarios y cumplir con sus compromisos y, más aún, con comprometerse en proyectos comunes.; y, por otro, con que la vida se vuelve impredecible. Es decir, en los intercambios sociales que se producen en estos contextos no se puede tener la certeza de que las interacciones son seguras y eficientes, aspectos estos que cumplen las reglas morales. Todas estas características estarían alimentando ciclos de violencia en estas sociedades.

Queda como tarea para futuros estudios, por un lado, explorar los cambios en el desarrollo con respecto a cómo los individuos razonan y evalúan actos de venganza tanto en poblaciones en condiciones normativas como no normativas. Por otro lado, sería importante explorar diferentes condiciones socioeconómicas ya que como se expuso, la violencia en países con conflictos armados prolongados se extiende a muchas esferas y en general todos los ciudadanos quedan expuestos de alguna manera a actos violentos. La evidencia presentada soporta la idea de que la exposición a violencia está relacionada con maneras de razonar acerca de situaciones sociomorales, más allá de que los individuos muestren o no síntomas psicológicos.

\section{Referencias}

Alvarado, S. V., Ospina, H. B. \& Luna, M. T. (2005). Concepciones de justicia en niños y niñas que habitan en contextos urbanos violentos. Revista Lati- noamericana de Ciencias Sociales, Niñez y Juventud, 3, 2-30. Disponible en http://redalyc.uaemex.mx/ redalyc/pdf/773/77330207.pdf

Astor, R. (1994). Children's moral reasoning about family and peer violence: The role of provocation and retribution. Child Development, 65, 1054-1067.

Astor, R. \& Behre, W. (1997). Violent and nonviolent children's and parents' reasoning about family and peer violence. Behavioral Disorders, 22, 231-245.

Bandura, A. (1991). Social cognitive theory of moral thought and action. En W. M. Kurtines \& J. L. Gewirtz (Eds.), Handbook of moral behavior and development: Theory, research and applications (Vol. 1, pp. 71-129). Hillsdale, NJ: Erlbaum.

Bandura, A., Barbaranelli, C., Caprara, G. V. \& Pastorelli, C. (1996). Mechanisms of moral disengagement in the exercise of moral agency. Journal of Personality and Social Psychology, 71, 364-374.

Barkin, S., Kreiter, S. \& DuRant, H. (2001). Exposure to violence and intentions to engage in moralistic violence during early adolescence. Journal of Adolescence, 24, 777-789.

Baumeister, R. (2000). Evil: Inside human violence and cruelty. New York: W. H. Freeman \& Company.

Buka, S. L., Stichick, T., Birdthistle, I. \& Earls, F. (2001). Youth exposure to violence: Prevalence, risks and consequences. American Journal of Orthopsychiatry, 71, 298-310.

Buckner, J. C., Beardslee, W. R. \& Bassuk, E. L. (2004). Exposure to violence and low-income children's mental health: Direct, moderated and mediated relations. American Journal of Orthopsychiatry, 74, 413-423.

Cairns, E. (1987). Caught in crossfire: Children and the Northern Ireland conflict. Belfast, Ireland: Appletree Press.

Coles, R. (1986). The political life of children. Boston: Atlantic Monthly Press.

Dawes, A. (1994). The effects of political violence on socio-moral reasoning and conduct. En A. Dawes $\&$ D. Donald (Eds.), Childhood and adversity_(pp. 200-219). Cape Town, South Africa: David Philip Publishers.

Echavarría Grajales, C. V. \& Vasco Montoya, E. (2006). Justificaciones morales de lo bueno y lo malo en un grupo de niños y niñas provenientes de con- 
textos violentos y no violentos de una ciudad de la zona andina de Colombia. Acta Colombiana de Psicología, 9, 51-62.

Echenique, C., Medina, L. M., Medina, A. R. \& Ramírez, A. (2008). Prevalencia del trastorno por estrés postraumático en población desplazada por violencia, en proceso de restablecimiento en Sincelejo. Psicología desde el Caribe, junio, 122-135.

Farver, J. A. \& Frosch, D. L. (1996). L. A. stories: Aggression in preschoolers' spontaneous narratives after the riots of 1992. Child Development, 67, 19-32.

Ferguson, N. \& Cairns, E. (1996). Political violence and moral maturity in Northern Ireland. Political Psychology, 17, 713-725.

Fitzpatrick, K. M. (1993). Exposure to violence and presence of depression among low-income AfricanAmerican youth. Journal of Consulting and Clinical Psychology, 61, 528-531.

Fonagy, P. (2003). Towards a developmental understanding of violence. British Journal of Psychiatry, 183, 190-192.

Franco, S. (1997). Violencia y salud en Colombia. Revista Panamericana de Salud Pública, 1, 93-103.

Fremont, W. (2004). Childhood reactions to terrorisminduced trauma: A review of the past 10 years. Journal of the American Academy of Child $\mathcal{E}$ Adolescent Psychiatry, 43, 381-392.

Gorman-Smith, D. \& Tolan, P. (1998). The role of exposure to community violence and developmental problems among inner-city youth. Development and Psychopathology, 10, 101-116.

Guerra, N. G., Huesmann, L. R. \& Spindler, A. (2003). Community violence exposure, social cognition, and aggression among urban elementary school children. Child Development, 74, 1561-1576.

Harpham, T., Grant, E. \& Rodríguez, C. (2004). Mental health and social capital in Cali, Colombia. Social Science and Medicine, 58, 2267-2278.

Helwig, C. C. (1995). Adolescents' and young adults' conceptions of civil liberties: Freedom of speech and religion. Child Development, 66, 152-166.

Helwig, C. C. (1997). The role of agent and social context in judgments of freedom of speech and religion. Child Development, 68, 484-495.
Helwig, C. C. (1998). Children's conceptions of fair government and freedom of speech. Child Development, 69, 518-531.

Herrman, H., Saxena, C. \& Moody, R. (Eds.). (2005). Promoting mental health: Concepts, emerging evidence, practice. Geneva: World Health Organization.

Horn, J. \& Trickett, P. (1998). Community violence and child development: A review of research. En P. Trickett \& C. Schellenbach (Eds.), Violence against children in the family and the community (pp. 103138). Washington, DC: American Psychological Association.

Internal Displacement Monitoring Centre. (2008). Rates of new displacement highest in two decades. Oct. 17, 2008. Recuperado en enero 2, 2009, de http://www. internal-displacement.org/8025708F004CE90B/ (httpCountries)/CB6FF99A94F70AED802570A 7004CEC41?OpenDocument

Jenkins, E. \& Bell, C. (1997). Exposure and response to community violence among children and adolescents. En J. D. Osofsky (Ed.), Children in a violent society (pp. 9-31). New York: The Guilford Press.

Keyes, C. L. M. (2003). Complete mental health: An agenda for the $21^{\text {st }}$ century. En C. L. M. Keyes \& J. Haidt (Eds.), Florishing: Positive psychology and the life well-lived (pp. 293-312). Washington DC: APA.

Kohlberg, L. (1976). Moral stages and moralization. En T. Lickona (Ed.), Moral development and behavior: Theory, research, and social issues (pp. 31-53). New York: Holt, Rinehart \& Winston.

Kuo, M., Mohler, B., Raudenbush, S. \& Earls, F. (2000). Assessing exposure to violence using multiple informants: Application of hierarchical linear model. Journal of Child Psychology and Psychiatry, 41, 1049-1056.

Laplante, L. J. \& Rivera Holguín, M. (2006). The Peruvian Truth Comission's mental health reparations: Empowering survivors of political violence to impact public health policy. Health and Human Rights, 9, 136-163.

Liddell, C., Kvalsvig, J., Qotyana, P. \& Shabalala, A. (1994). Community violence and young South African children's involvement in aggression. International Journal of Behavioral Development, 17, 613-628. 
Londoño, N. H., Muñiz, O., Correa, J. E., Patiño, C. D., Jaramillo, J., Raigoza, J. et al. (2005). Salud mental en víctimas de la violencia armada en Bojayá (Chocó, Colombia). Revista Colombiana de Psiquiatría, 34, 493-505.

Lorenc, L. \& Branthwaite, A. (1986). Evaluations of political violence by English and Northern Irish school children. British Journal of Social Psychology, 25, 349-352.

Lynch, M. (2001, abril). The unique and combined effects of community violence and child maltreatment on children's adaptation. Paper presented at the Biennial Meeting of the Society for Research in Child Development, Minneapolis, Minnesota, United States.

Lynch, M. \& Cicchetti, D. (1998). An ecological-transactional analysis of children and contexts: The longitudinal interplay among child maltreatment, community violence, and children symptomatology. Development and Psychopathology, 10, 235-257.

Macksoud, M. \& Aber, L. (1996). The war experiences and psychological development of children in Lebanon. Child Development, 67, 70-88.

Mansour, S. (2002). A week in Jenin: Assessing mental health needs amid the ruins. Journal of Palestine Studies, 4, 35-43.

Martinez, P. \& Richters, J. E. (1993). The NIMH community violence project: II. Children's distress symptoms associated with violence exposure. Psychiatry, 56, 22-35.

Médecins Sans Frontières. (2006). Living in fear: Colombia's cycle of violence. Disponible en https:// www.msf.org.au/docs/reports/colombia_living_ in_fear_0406.pdf

Miller, L. S., Wasserman, G. A., Neugebauer, R., Gorman-Smith, D. \& Kamboukos, D. (1999). Witnessed community violence and antisocial behavior in high-risk, urban boys. Journal of Clinical Child Psychology, 28, 2-11.

Osofsky, J. D., Wewers, S., Hann, D. M. \& Fick, A. C. (1993). Chronic community violence: What is happening to our children? Psychiatry, 56, 36-45.

Palacio, J., Sabatier, C., Abello, Ll. R. \& Madariaga, O. C. (2001, abril). Impact of political violence and displacement on Colombian children. Poster presentado en el Encuentro Bianual de la Society for Research in Child Development, Minneapolis, Minnesota, United States.

Pérez-Olmos, I., Fernández-Piñeres, P. E. \& RodadoFuentes, S. (2005). Prevalencia del trastorno por estrés postraumático por la guerra, en niños de Cundinamarca, Colombia. Revista de Salud Pública, 7, 268-280.

Posada, R. (2008). Making sense of interpersonal conflicts from a victim and a perpetrator perspective: Colombian displaced adolescents tell about their social experiences. Disertación de Doctorado, University of Utah, Salt Lake City, United States.

Posada, R. (2009, abril). Trying to restore justice: Exposure to violence and vengeance. Poster presentado en la conferencia del Biennial Meeting of the Society for Research in Child Development, Denver, United States.

Posada, R. \& Wainryb, C. (2006, junio). Exposure to violence, morality, and revenge. Poster presentado en el 34th Symposium of the Jean Piaget Society, Baltimore, United States.

Posada, R. \& Wainryb, C. (2008). Moral development in a violent society: Colombian children's judgments in the context of survival and revenge. Child Development, 79, 882-898.

Punamäki, R. L. (1996). Can ideological commitment protect children's psychosocial well-being in situations of political violence? Child Development, 67, 55-69.

Pynoos, R. S., Frederick, C., Nader, K. \& Arroyo, W. (1987). Life threat and posttraumatic stress in school-age children. Archives of General Psychiatry, 44, 1057-1063.

Quintero Mejía, M. \& Vasco Montoya, E. (2007). Justificaciones y sentimientos morales de jóvenes universitarios y jóvenes desplazados acerca de las acciones justas e injustas. Acta Colombiana de Psicología, 10, 99-110.

Richters, J. E. \& Martinez, P. (1993). The NIMH community violence project: I. Children as victims of and witnesses to violence. Psychiatry, 56, 7-21.

Rodríguez, J., De la Torre, A. \& Miranda, C. T. (2002). La salud mental en situaciones de conflicto armado. Biomédica, 22, 337-346.

Sanderson, A. (2001). A narrative analysis of behaviorally troubled adolescents' life stories (Doctoral 
dissertation, The University of Calgary, 2001). Dissertation Abstracts International, 62, 4076.

Selner-O'Hagan, M. B., Kindlond, D. J., Buka, S. L., Raudenbush, S. W. \& Earls, F. J. (1998). Assessing exposure to violence in urban youth. Journal of Child Psychology and Psychiatry, 39, 215-224.

Shaw, L. \& Wainryb, C. (1999).The outsider's perspective: Young adults' judgments of social practices of other cultures. British Journal of Developmental Psychology, 17, 451-471.

Smetana, J. G. (1995). Morality in context: Abstractions, ambiguities, and applications. En R. Vasta (Ed.), Annals of child development (Vol. 10, pp. 83 130). Londres: Jessica Kingsley.

Smetana, J. G., Kelly, M. \& Twentymen, C. T. (1984). Abused, neglected, and nonmaltreated children's conceptions of moral and social-conventional transgressions. Child Development, 55, 277-287.

Tisak, M. S. \& Block, J. H. (1990). Preschool children's evolving conceptions of badness: A longitudinal study. Early Education and Development, 1, 300 . 307.

Turiel, E. (1983). The development of social knowledge: Morality and convention. Cambridge: Cambridge University Press.

Turiel, E. (1998). The development of morality._En W. Damon \& N. Eisenberg (Eds.), Handbook of child psychology. Social, emotional and personality development (Vol. 3, pp. 863-932). New York: John Wiley \& Sons.

Wainryb, C. (1991). Understanding differences in moral judgments: The role of informational assumptions. Child Development, 62, 840-851.

Wainryb, C. (1993). The application of moral judgments to other cultures: Relativism and universality. Child Development, 64, 924-933.

Wainryb, C., Brehl, B. \& Matwin, S. (2005). Being hurt and hurting others: Children's narrative accounts and moral judgments of their own interpersonal conflicts. Monographs of the Society for Research in Child Development, 70 (3, Serial No. 281).

Wainryb, C. \& Ford, S. (1998). Young children's evaluations of acts based on beliefs different from their own. Merrill-Palmer Quarterly, 44, 484-503.

Wainryb, C., Shaw, L. \& Maianu, C. (1998). Tolerance and intolerance: Children's and adolescents' judgments of dissenting beliefs, speech, persons, and conduct. Child Development, 69, 1541-1555.

Walton, M. D. \& Brewer, C. L. (2001). The role of personal narrative in bringing children into the moral discourse of their culture. Narrative Inquiry, 11, 307-334. 
